\title{
Psychological health and HIV transmission amongst female sex workers:
}

\section{A systematic review}

Winnie Wing-Yan Yuen, Department of Family Medicine and Primary Care, The University of Hong Kong, Hong Kong. 3/F., Ap Lei Chau Clinic, 161 Main Street, Ap Lei Chau, Hong Kong. Telephone: (852)2553-4514. Fax: (852) 2814-7475. Email address: winnie.yuen@hku.hk

Lynn Tran, Department of Family Medicine and Primary Care, The University of Hong Kong, Hong Kong.

Carlos King-Ho Wong, Department of Family Medicine and Primary Care, The University of Hong Kong, Hong Kong.

Eleanor Holroyd, School of Health Sciences, RMIT University, Melbourne, Victoria, Australia.

Catherine So-kum Tang, Department of Psychology, Faculty of Arts and Social Sciences, National University of Singapore, Singapore.

William Chi-Wai Wong, Department of Family Medicine \& Primary Care, The University of Hong Kong, Hong Kong. 


\title{
Psychological Health and HIV Transmission amongst Female Sex Workers:
}

\section{A Systematic Review and Meta-analysis}

\author{
Abstract \\ Current HIV prevention interventions for female sex workers (FSWs) have tended to \\ target the cognitive factors in changing their behaviors, yet little attention has been paid \\ to the psychological factors that influence the behavior of women in sex work. This \\ review aimed to explore the associations between the psychological health of FSWs \\ and HIV risk. A total of 8 studies published in English before July 2013 were reviewed. \\ Studies showed that FSWs had reported more psychological issues, including \\ depression, suicidal thoughts and lower quality of life, and the pooled prevalence of \\ probable depression were as high as $62.4 \%$. Majority of studies showed that higher \\ scores in psychological health problems were associated with increased HIV risk \\ behaviors, in particular inconsistent condom use, or STI infections. Among the five \\ studies which measured symptoms of depression, four documented that higher \\ depression scores were significantly associated with inconsistent condom use among \\ FSWs with their clients and/or partners. Meta-analysis was performed using a fixed \\ effects model to examine the association between depression and inconsistent condom \\ use and found higher scores in depression were significantly associated with \\ inconsistent condom use (odds ratio $=2.57, \mathrm{p}<0.001$ ). This review contends that future \\ HIV preventive interventions should take psychological health of FSWs into \\ consideration.
}


Keywords: HIV prevention; harm reduction; prostitution; psychological health; risk behaviors

Word count: 205 


\section{Introduction}

Poor psychological health can act as a precursor of health risk behaviors through the interactions of thoughts, feelings and behaviors. Sexual risk behaviors are one of the health risks that associated with psychological disorders (Pearson et al., 2008; Turner, Latkin, Sonenstein, \& Tandon, 2011); for example, women with psychotic disorders were less likely to engage in consistent condom use (Weinhardt, Carey, Carey, Maisto, \& Gordon, 2001; Weinhardt, Carey, Carey, \& Verdecias, 1998). A longitudinal study reported that depressive symptoms were associated with poor condom use, alcohol use or substance use and multiple sexual partners among adolescents (Lehrer, Shrier, Gortmaker, \& Buka, 2006). A meta-analytic study showed that women with multiple depressive symptoms were more likely to report unprotected sex and more life-time sexual partners (Logan, Cole, \& Leukefeld, 2002; Orr, Celentano, Santelli, \& Burwell, 1994).

The association between psychological health and HIV risks could be explained by cognitive model (Beck, 1967), in which maladaptive thoughts influence one’s emotions and behaviors. For instance, individuals with depression often have negative thoughts about themselves, the world and their future, which potentially reduce motivation to care for themselves and increase propensity to engage in HIV risk behavior. Moreover, negative emotions, such as anger and fear may interfere how one perceives their sexual risks. In a systematic review, the 20 identified articles showed associations between negative affect and HIV risks; however, the meta-analysis did not support the relationship statistically (Crepaz \& Marks, 2001). However, it has been argued the 
insignificant result may be due to methodological limitations that heterogeneous populations with different HIV risks, such as heterosexual men and men who have sex with men, were included in the study (Kalichman \& Weinhardt, 2001).

Female sex workers (FSWs), with few choices upon entering into this occupation, are vulnerable to contracting HIV because of the associated greater number of sexual partners, mobility, and inconsistent condom and drug use (El-Bassel, Witte, Wada, Gilbert, \& Wallace, 2001). This has placed them as one of the key populations for HIV research and prevention to control the spread of infection. Global spending on HIV responses has increased to US\$18.9 billion in 2012 with 19\% on prevention alone (UNAIDS, 2013). It is further estimated that funding for HIV programs needs to be increased globally to US $\$ 22.0$ billion by 2015 to achieve universal access to HIV prevention, treatment and care (UNAIDS, 2011). Therefore, identifying risk factors contributing to HIV risk behaviors, such as unprotected sex, is a vital step to develop effective HIV prevention interventions (Platt et al., 2013).

\section{Risk factors of HIV infection among FSWs}

The HIV risk factors among FSWs can be generally divided into environmental and individual factors. The former includes poverty, mobility, social disruption (Parker, Easton, \& Klein, 2000), sexual culture (Basuki et al., 2002; Y. Hong \& Li, 2008), stigma (Wong, Holroyd, \& Bingham, 2011) and poor working conditions (Agha \& Chulu Nchima, 2004; Scambler \& Paoli, 2008; Yi et al., 2010), especially the behaviors of the brothel managers (Y. Hong \& Li, 2008; Kerrigan et al., 2003). Individual factors, such as sexual history, sex work experience, poor HIV knowledge (Yi et al., 2010), history of abuse, and low level of self-efficacy (Larios et al., 2009) were also found to 
be related to increased HIV risks. For example, women who had a history of sexual abuse or victimization were more likely to engage in unprotected sex or inappropriate condom use (Lang, Salazar, DiClemente, \& Markosyan, 2013; Logan et al., 2002; Zhang et al., 2012).

Sex work is a highly marginalized occupation with a unique set of health risks (Scambler \& Paoli, 2008; Wong et al., 2011). Many women enter sex work due to financial difficulties and family problems (Vanwesenbeeck, 2001). Limited social capital, self-stigma and public stigma have often predisposed them to a variety of psychological vulnerabilities (Yan Hong et al., 2009). These problems may be further intensified by workplace violence, and the risk of HIV/sexually transmitted infections (STIs). Research findings revealed that depression, helplessness, anxiety, lower selfworth, and quality of life were commonly reported among sex workers (El-Bassel et al., 1997; Gorry, Roen, \& Reilly, 2010; Wong, Holroyd, Gray, \& Ling, 2006). As mentioned before, high level of psychological distress was related to increased risks to contracting HIV. However, such associations among FSWs have yet been confirmed in the literature.

The current study aimed to examine whether FSW's who had poor psychological health had increased likelihood of inconsistent condom use, a crucial link in HIV prevention programs. This paper described the characteristics of these studies, primarily to explore associations between psychological health of FSWs and HIV risk behaviors. The study further highlighted the research gaps in existing literature to provide recommendations for future direction of HIV prevention. 


\section{Methods}

\section{Materials}

A comprehensive literature search was conducted using four databases: EMBASE, ISI Web of Science, PsycInfo and PubMed in July 2013. The PRIMSA 2009 checklist was used to guide the systematic review process. The inclusion criteria were quantitative peer-reviewed studies that: (1) primarily focused on FSWs; (2) reported any psychological health outcomes from disorder-specific measures to global measures of psychological well-being; (3) included at least one dependent variable on HIV infection rate or HIV risks, such as condom use and HIV testing; and, (4) examined the relationship between psychological health and HIV risk using multivariable methods. Articles were limited to those published in English on or before July 2013. We excluded any grey literature including government reports, non-published papers, doctoral theses, or those without original data. Studies that sampled male sex workers, transgender sex workers, or without reporting separate FSWs data were also excluded. Studies concerning alcohol and drug use were excluded in this review because there is already a recent literature review published for alcohol use and HIV risks (Li, Li, \& Stanton, 2010). Substance use encompasses a wide range of illicit and non-illicit drugs, and to disentangle its associations with HIV risks and other psychological health outcomes and disorders is beyond the scope of this review.

In order to identify as many papers as possible, we initially searched for studies that examined all risk factors to HIV risk behaviors and then screened for those related to psychological health. The following key words were used: "sex workers”, “prostitutes”, “prostitution” AND “risk factors” or “correlates”, AND “HIV” or “condom use” or "safe sex”. Cited publications in the reference list of retrieved articles 
were then screened manually for additional relevant studies. After titles and abstracts that fulfilled all inclusion criteria were screened, full-text articles were obtained for further assessment by two independent reviewers. The final list of articles to be reviewed was discussed among the two reviewers and any disagreements on inclusion were resolved by discussion with the third independent reviewer.

\section{Data extraction and analysis}

Two reviewers (WY and LC) independently extracted data from the articles within a number of predefined categories, including the place where the study was conducted, year conducted, study design, sample size, inclusion criteria of participants, method of analyses, psychological health outcomes, rate of condom use, rate of HIV/AIDS, and strengths and weaknesses of each study (Moher, Liberati, Tetzlaff, \& Altman, 2009). In accordance with the primary aim of the investigation, the relationships between psychological health and HIV risk behaviors among FSWs were also included. Several studies measured psychological disorder symptoms using scales with different cut-off points, such as the Center for Epidemiologic Studies Depression Scale (Radloff, 1977), for predicting a variety of HIV risk behaviors (e.g. inconsistent condom use in past 7 days, 6 months or without specifying the time, condom use error). The pooled prevalence of psychological problem measured by the studies was calculated using the event rates and total number of participants of available studies. For studies that included a regression model of psychological symptoms and inconsistent condom use were further examined for meta-analysis. The odds ratio (OR) for inconsistent condom use, as well as its lower limit and upper limit of individual study were integrated with the meta-analysis software Comprehensive Meta-Analysis Version 2.2.064 (Biostat, Inc, 
Tampa, FL). The pooled estimate of OR was calculated and analysed using a fixedeffect model.

\section{Results}

A total of 3,885 articles were identified from the databases and 17 were obtained from the references of the articles. Of these, 1,188 articles were excluded due to duplication and 2,464 were excluded as the abstracts did not fulfil the inclusion criteria. The remaining 250 studies were examined in full-text against the inclusion criteria, 242 were further excluded for reasons specified in Figure 1. A total of eight original articles were subsequently included in this review.

(Figure 1 to be inserted here)

The quality of the papers was assessed with regards to the sampling methods, outcome measures, statistical tests used. All the reviewed articles addressed a focused issue which was clearly stated in the introduction and the inclusion criteria were reported in all studies. Different sampling methods were employed, including convenience sampling (four studies), ethnographic targeted sampling (one study), multi-stage cluster probability sampling (two studies), and stratified random sampling (one study). Five out of eight studies reported the response rate (from $80 \%$ to $98.9 \%$ ). All studies using interviewers to gather data reported that they receive relevant training. A majority of the studies used validated scales or diagnostic criteria to measure the psychological health of participants. The statistical tests used in individual study were 
described clearly in all papers.

\section{Characteristics of studies}

All studies reviewed were cross-sectional in design. The sample populations were recruited from a range of institutions such as brothels, street, indoor entertainment establishments. Study sites included China (e.g. Guangxi and Hong Kong), India, Korea, the Philippines, Puerto Rico, Armenia, and the US (Table 1). Most studies collected information through self-administered questionnaires, face-to-face interviews, and four studies had undertaken blood tests or vaginal swabs from participants for HIV and/or STI testing (Alegria et al., 1994; Jung, 2012, 2013; Shahmanesh et al., 2009). All study explored the associations of psychological health and HIV risk behaviors among FSWs using regression models. In most studies, a number of psychological variables were put into the regression models to determine the independent predictors on different HIV risk behaviors as proxy measures of HIV transmission, including inconsistent condom use, condom use errors and the presence of HIV/STI infections.

(Table 1 to be inserted here)

\section{Psychological health of FSWs}

One of the most commonly reported psychological issues was depression. Among the studies that used CES-D with the cut-off score of 16 (Alegria et al., 1994; Y. Hong, Li, Fang, \& Zhao, 2007; Lau, Tsui, Ho, Wong, \& Yang, 2010), 53.9\% to 70.1\% of FSWs interviewed had high levels of depressive symptoms. In one study, Urada et al. found that $78 \%$ of women had higher levels of depressive symptoms with the cut-off score of 
23 (Urada, Morisky, Hernandez, \& Strathdee, 2013). The pooled prevalence of probable depression was calculated based on these four studies was 62.4\% $(\mathrm{p}<0.001)$. Figure 2 shows the forest plot of the pooled prevalence from these studies.

(Figure 2 to be inserted here)

Apart from depression, other psychological variables included suicidal ideation, and suicidal attempts (Jung, 2012, 2013; Shahmanesh et al., 2009), quality of life, social isolation or social support among FSWs (Jung 2012; Shahmanesh et al., 2009; Urada, 2013). It has shown that $18.7 \%$ to $28.3 \%$ of FSWs attempted suicide (Jung, 2012, 2013; Shahmanesh et al., 2009) while one study found 37.7\% of women had suicidal ideation (Lau et al., 2010). In terms of overall psychological well-being, a study found that $25 \%$ and $22.3 \%$ of the Chinese FSWs interviewed had low quality of life and life satisfaction respectively (Lau et al., 2010).

\section{Inconsistent condom use and HIV infections}

The rate of inconsistent condom use with FSWs' clients ranged from 25.6\% (Shahmanesh et al., 2009) to 62.5\% (Hong et al., 2007). This variation range of inconsistent condom use could be influenced by each study's definition of the question; however, not all studies specified it. One study reported that $78.0 \%$ of interviewees reported condom use "errors" as measured by penetration without a condom, condom being taken off before sex was over, condom breakage, condom slipped off and/or condom became dry during sex (Lang, Salazar, DiClemente, Markosyan, \& Darbinyan, 2011). As for HIV infection, only three studies reported the HIV status of the 
participants. Of note one study showed no positive case of HIV infection (Jung, 2012), whereas one showed that the incidence rate was $4.7 \%$ per annum with a prevalence of 25.7\% (Shahmanesh et al., 2009). Furthermore, another study found confirmed HIV rate at 32.1\% (Alegria et al., 1994).

\section{Psychological Health and HIV risks}

Among the studies that had used regression models to investigate the relationship between psychological health and HIV risk, only two (Shahmanesh et al., 2009; Urada et al., 2013) showed no significant association with inconsistent condom use or STIs. Overall, the great majority of these studies $(\mathrm{xx} / \mathrm{xx})$ found that the lower the scores in psychological health variables, the higher rate of inconsistent condom use or STI infections. Among that five studies had used CES-D, four of them found higher depression scores or presence of depression symptoms were significantly associated with inconsistent condom use with clients and/or partners (Alegria et al., 1994; Y. Hong et al., 2007; Lang et al., 2011; Lau et al., 2010). In one of these studies, the authors used separate models for predicting unprotected vaginal and oral sex with clients, in that FSWs with higher levels of depressive symptoms were twice as likely to engage in unprotected oral sex with clients (Lau et al., 2010).

A meta-analysis was conducted in the four studies that had used regression models to investigate the relationshipa between depressive symptoms and condom use. Figure 3 shows the Forest plot for the associations between depression and inconsistent condom use during vaginal intercourse with clients. The overall depressive symptoms was significantly correlated with inconsistent condom use: FSWs who had a higher depressive symptoms as measured by CES-D were about 2.5 times more likely to use 
condom inconsistently than those who reported a lower depressive symptoms $(\mathrm{OR}=$ $2.57,95 \% \mathrm{CI}=1.87-3.54, p<0.001)$.

Other psychological health measures reported included suicidal ideation, lower social support, being pessimistic about the future, hopelessness, and being victimised, These factors were found to be significantly associated with inconsistent use of condom or HIV infections. In particular, three studies explored the association of suicidal attempts and condom use or HIV/STI status, while one study conducted in Korea found that suicidal attempts were significantly associated with inconsistent condom use (Jung, 2013); two others showed that suicidal attempts were related to STI infections (Jung, 2012; Shahmanesh et al., 2009). However, a study conducted in India failed to find the association of deliberate self-harm or mental health score and HIV infection (Shahmanesh et al., 2009). At the same time, other psychological variables seem to play a role in raising their risks of HIV. FSWs who reported to be pessimistic were more likely to use condom inconsistently (Lau et al., 2010), while those who had less social support was associated with HIV infection (Shahmanes et al., 2009). These findings suggested that poor psychological health could increase HIV risks among FSWs.

\section{Discussion}

The current systematic review includes eight studies with FSWs from brothels, street and other entertainment establishments across nine countries. Psychological vulnerabilities were reported by FSWs, including depressive symptoms, suicidal thoughts and attempts, and poor general psychological wellbeing. Also, psychological 
problems are significant predictors of HIV risk behaviors in the great majority of the studies under review, in particular self-reported depressive symptoms are shown to be related to inconsistent condom use in a meta-analysis.

Unlike previous meta-analyses (Crepaz \& Marks, 2001), this review suggests that psychological problems and inconsistent condom use are indeed related. The difference in the findings between this and previous reviews highlights that FSWs are a unique group of women with a discrete set of psychosocial risks (e.g. stigma, violence) and resources compared to other at-risk populations. We contend that investigations of the factors on HIV risk, behaviors and preventive measures should be conducted in individual at-risk populations.

While current HIV prevention programs based on health psychology theories have traditionally used the concepts of threat of disease to reduce risk behaviors, the emotional state of the target population are seldom addressed. Over half of the studies in this review have shown that poor psychological health as measured by depressive symptoms and suicidal attempts of FSWs predict inconsistent condom use. According to Beck’s cognitive theory (Beck, 1967), it is possible that FSWs would have negative thoughts and helplessness about their work and themselves. Other studies supported this proposition and showed that FSWs who expressed feelings pessimistic about their life and future (Lau et al., 2010) were more likely to engage in HIV risk behaviors. Furthermore, FSWs' perception of their ability and self-control over an event was associated with their mood state, which appeared to play a role in HIV risk behaviors (Y. Hong et al., 2007). It is argued that, in the presence of depressive symptoms, it may affect FSWs’ perceived control over a sexual encounter, and hence a lower 
predisposition to use condom with their clients. We therefore recommend that future interventions for FSWs should take into account their psychological health and especially their negative emotions.

This review also highlights some research gaps in the understanding of HIV risks among FSWs, giving rise to implications for future research. There was a relatively small number of research papers explored both the psychological health of FSWs and HIV risk behaviors, and many studies were excluded from the current review because they did not conduct statistical analysis to investigate such relationship. Further, over-emphasis of Health Belief Model (Rosenstock, 1974), Theory of Reasoned (Ajzen, 1985) and Theory of Planned Behavior (Ajzen \& Fishbein, 1980) by many scholars in this field, leads to the neglect of important emotional factors in HIV prevention. Systematic reviews based on these cognitive and behavioral models on the effectiveness of HIV prevention programs indeed found that harm prevention strategies (e.g. promoting condom use) that removing barriers, changing subjective norm, beliefs and attitudes, and targeted sexual health education are commonly used but "not always effective” (Catania, Kegeles, \& Coates, 1990; Morrison, Baker, \& Gillmore, 1998).

Thus far, all studies under review have been cross-sectional. It is difficult to ascertain if their psychological problems happened before, during or after engaging in sex work and how these problems interact with HIV risk behaviors. While a longitudinal prospective study would be ideal, the high degree of fluidity and mobility of this population pose considerable challenges (Y. Hong \& Li, 2008; Vanwesenbeeck, 2001). Other research directions should therefore investigate the processes by which the emotional status of FSWs influences risk behaviors or examine whether managing these 
emotional states could promote self-esteem and hence harm reduction behaviors. Such an in-depth understanding will help expand the psychological models of health behaviors.

Furthermore, a wide range of outcome measures were used making results comparison difficult. For risk behaviors such as inconsistent condom use, the definitions varred from "condom use in the last intercourse" to "unprotected vaginal sex with clients in the past one month”. Researchers may use the questionnaires and validated scales developed in the previous studies to measure behavioral and HIV risks. Lastly, since sex work is not legal in many regions, FSWs constituting a vulnerable and “hard-to-reach” population and mostly recruited through convenience sampling. To improve the rigour of future studies, we would recommend using more recently developed sampling methods such as respondent driven sampling to recruit participants.

There are several limitations of the current systematic review. First, a relatively small number of studies were included and many of which were conducted in Asian countries; therefore the results should be interpreted with cautions. Secondly, the rates of psychological issues reported only reflect a subset of the studies which investigated both psychological distress and risk behaviors. Despite these limitations, the association between psychological outcomes, in particular depressive symptoms, and HIV risk behaviors is convincing, indicating the need to take psychological health of FSWs into consideration when designing and implementing HIV prevention interventions. As suggested by some of the studies (Y. Hong et al., 2007), "HIV prevention programs for FSWs should be focused on identifying and treating psychological health issues to reduce unprotected sex and sexual health risks among FSWs”. 


\section{Conclusions}

In summary, the population of FSWs in these eight review articles had psychological issues including depression, suicidal ideation, and lower scores on quality of life and social support. In addition, their poor psychological health was shown to increase their likelihood to engage in inconsistent condom use. Research gaps identified from this study was limited research, especially longitudinal studies focusing on the link between psychological health and HIV risk behaviors. Understanding such association has significant implications on designing new HIV prevention programs for targeted at-risk populations. 


\section{References}

Agha, S., \& Chulu Nchima, M. (2004). Life-circumstances, working conditions and HIV risk among street and nightclub-based sex workers in Lusaka, Zambia. Culture, Health \& Sexuality, 6(4), 283-299. doi:10.1080/13691050410001680474

Ajzen, I. (1985). From intentions to actions: a theory of planned behavior. In J. Kuhl \& J. Beckman. (Eds.), Action-control: From cognition to Behavior. Heidelberg: Springer Verlag.

Ajzen, I., \& Fishbein, M. (1980). Understanding attitudes and predicting social behavior. Englewood Cliffs, N.J.: Prentice-Hall.

Alegria, M., Vera, M., Freeman Jr, D. H., Robles, R., Santos, M. d. C., \& Rivera, C. L. (1994). HIV infection, risk behaviors, and depressive symptoms among Puerto Rican sex workers. American Journal of Public Health, 84(12), 2000-2002.

Basuki, E., Wolffers, I., Devillé, W., Erlaini, N., Luhpuri, D., Hargono, R., . . . Beelen, N. v. (2002). Reasons For Not Using Condoms Among Female Sex Workers in Indonesia. AIDS Education and Prevention, 14(2), 102-116. doi:10.1521/aeap.14.2.102.23901

Beck, A. (1967). Depression: Clinical, experimental, and theoretical aspects. New York: Hoeber.

Beck, A., Steer, R., \& Brown, G. (1996). Manual for the BDI-II: San Antonio, TX: Psychological Corporation.

Catania, J. A., Kegeles, S. M., \& Coates, T. J. (1990). Towards an understanding of risk behavior: An AIDS risk reduction model (ARRM). Health Education Quarterly, 17(53-72).

Crepaz, N., \& Marks, G. (2001). Are negative affective states associated with HIV sexual risk behaviors? A meta-analytic review. Health Psychology, 20(4), 291-299. doi:10.1037/1040-3590.4.2.123

El-Bassel, N., Schilling, R. F., Irwin, K. L., Faruque, S., Gilbert, L., Von Bargen, J., . . . Edlin, B. R. (1997). Sex trading and psychological distress among women recruited from the streets of Harlem. American Journal of Public Health, 87(1), 66-70. doi:10.2105/ajph.87.1.66

El-Bassel, N., Witte, S. S., Wada, T., Gilbert, L., \& Wallace, J. (2001). Correlates of Partner Violence Among Female Street-Based Sex Workers: Substance Abuse, History of Childhood Abuse, and HIV Risks. AIDS Patient Care \& STDs, 15(1), 41-51. doi:10.1089/108729101460092

Gorry, J., Roen, K., \& Reilly, J. (2010). Selling your self? The psychological impact of street sex work and factors affecting support seeking. Health Soc Care Community, 18(5), 492-499. doi:10.1111/j.1365-2524.2010.00925.x

Gu, J., Lau, J. T., Chen, H., Chen, X., Liu, C., \& Liu, J. (2010). Mental health and interpersonal factors associated with HIV-related risk behaviors among noninstitutaionlized female injection drug users who are also sex workers in China. Women \& Health, 50, 20-36.

Hong, Y., Fang, X., Li, X., Liu, Y., Li, M., \& Tai-Seale, T. (2009). Self-perceived stigma, depressive symptoms, and suicidal behaviors among female sex workers in China. Journal of Transcultural Nursing.

Hong, Y., \& Li, X. (2008). Behavioral studies of female sex workers in China: A literature 
review and recommendation for future research. AIDS Behav, 12(4), 623-636.

Hong, Y., Li, X., Fang, X., \& Zhao, R. (2007). Depressive symptoms and condom use with clients among female sex workers in China. Sex Health, 4(2), 99-104.

Jung, M. (2012). Sexual, behavioral, and social characteristics of female sex workers and their risk of sexually transmitted infections: in South Korea. Sexuality and Disability, 30(4), 421-431.

Jung, M. (2013). Associations of Physical and Sexual Health with Suicide Attempts Among Female Sex Workers in South Korea. Sexuality and Disability, 31(3), 275286.

Kalichman, S. C., \& Weinhardt, L. (2001). Negative affect and sexual risk behavior: Comment on Crepaz and Marks (2001). Health Psychology, 20(4), 300-301.

Retrieved from http://search.proquest.com/docview/614367263?accountid=14548

http://library.hku.hk:4551/resserv?genre=article\&issn=02786133\&title $=$ Health+Psychology\&volume $=20 \&$ issue $=4$ \&date $=2001-07-$ 01\&atitle=Negative+affect+and+sexual+risk+behavior\%3A+Comment + on + Crep az+and+Marks+\%282001\%29.\&spage=300\&aulast=Kalichman\&sid=ProQ:ProQ \%3Apsycarticles\&isbn=\&jtitle=Health+Psychology\&btitle=

Kerrigan, D., Ellen, J. M., Moreno, L., Rosario, S., Katz, J., Celentano, D. D., \& Sweat, M. (2003). Environmental-structural factors significantly associated with consistent condom use among female sex workers in the Dominican Republic. AIDS, 17(3), 415-423. Retrieved from http://journals.lww.com/aidsonline/Fulltext/2003/02140/Environmental_structural factors_significantly.16.aspx

Lang, D., Salazar, L., DiClemente, R., \& Markosyan, K. (2013). Gender Based Violence as a Risk Factor for HIV-Associated Risk Behaviors Among Female Sex Workers in Armenia. AIDS Behav, 17(2), 551-558. doi:10.1007/s10461-012-0245-7

Lang, D., Salazar, L., DiClemente, R., Markosyan, K., \& Darbinyan, N. (2011). Predictors of condom errors among sex workers in Armenia. International journal of STD \& AIDS, 22(3), 126-130.

Larios, S. E., Lozada, R., Strathdee, S. A., Semple, S. J., Roesch, S., Staines, H., . . . Patterson, T. L. (2009). An exploration of contextual factors that influence HIV risk in female sex workers in Mexico: The Social Ecological Model applied to HIV risk behaviors. AIDS Care, 21(10), 1335-1342. doi:10.1080/09540120902803190

Lau, J. T., Tsui, H. Y., Ho, S. P., Wong, E., \& Yang, X. (2010). Prevalence of psychological problems and relationships with condom use and HIV prevention behaviors among Chinese female sex workers in Hong Kong. AIDS Care, 22(6), 659-668. doi:10.1080/09540120903431314

Lehrer, J. A., Shrier, L. A., Gortmaker, S., \& Buka, S. (2006). Depressive symptoms as a longitudinal predictor of sexual risk behaviors among US middle and high school students. Pediatrics, 118(1), 189-200.

Li, Q., Li, X., \& Stanton, B. (2010). Alcohol use among female sex workers and male clients: an integrative review of global literature. Alcohol and Alcoholism, 45(2), 188-199.

Logan, T., Cole, J., \& Leukefeld, C. (2002). Women, sex, and HIV: social and contextual factors, meta-analysis of published interventions, and implications for practice 
and research. Psychological Bulletin, 128(6), 851.

Lovibond, P. F., \& Lovibond, S. H. (1995). The structure of negative emotional states: Comparison of the Depression Anxiety Stress Scales (DASS) with the Beck Depression and Anxiety Inventories. Behaviour research and therapy, 33(3), 335343.

Moher, D., Liberati, A., Tetzlaff, J., \& Altman, D. G. (2009). Preferred Reporting Items for Systematic Reviews and Meta-Analyses: The PRISMA StatementThe PRISMA Statement. Annals of Internal Medicine, 151(4), 264-269. doi:10.7326/0003-4819-151-4-200908180-00135

Morrison, D. M., Baker, S. A., \& Gillmore, M. R. (1998). Condom use among high-risk heterosexual teens: A longitudinal analysis using the theory of reasoned action. Psychology \& Health, 13(2), 207-222. doi:10.1080/08870449808406747

Orr, S. T., Celentano, D. D., Santelli, J., \& Burwell, L. (1994). Depressive symptoms and risk factors for HIV acquisition among Black women attending urban health centers in Baltimore. AIDS Education and Prevention.

Parker, R. G., Easton, D., \& Klein, C. H. (2000). Structural barriers and facilitators in HIV prevention: a review of international research. AIDS, 14 Suppl 1, S22-32.

Pearson, F. S., Cleland, C. M., Chaple, M., Hamilton, Z., Prendergast, M. L., \& Rich, J. D. (2008). Substance Use, Mental Health Problems, and Behavior at Risk for HIV: Evidence from CJDATS. Journal of Psychoactive Drugs, 40(4), 459-469. doi:10.1080/02791072.2008.10400652

Platt, L., Jolley, E., Rhodes, T., Hope, V., Latypov, A., Reynolds, L., \& Wilson, D. (2013). Factors mediating HIV risk among female sex workers in Europe: a systematic review and ecological analysis. BMJ Open, 3(7). doi:10.1136/bmjopen-2013002836

Radloff, L. S. (1977). The CES-D scale a self-report depression scale for research in the general population. Applied psychological measurement, 1(3), 385-401.

Rosenstock, I. M. (1974). The health belief model and preventive health behaviour. Health Education Monographs, 2, 354-386.

Scambler, G., \& Paoli, F. (2008). Health work, female sex workers and HIV/AIDS: Global and local dimensions of stigma and deviance as barriers to effective interventions. Social Science \& Medicine, 66(8), 1848-1862. doi:http://dx.doi.org/10.1016/j.socscimed.2008.01.002

Shahmanesh, M., Wayal, S., Cowan, F., Mabey, D., Copas, A., \& Patel, V. (2009). Suicidal behavior among female sex workers in Goa, India: The sildent Epidemic. American Journal of Public Health, 99(7), 1239-1246. doi:10.2105/ajph

Surratt, H. L., Kurtz, S. P., Chen, M., \& Mooss, A. (2012). HIV risk among female sex workers in Miami: the impact of violent victimization and untreated mental illness. AIDS Care, 24(5), 553-561.

Surratt, H. L., Kurtz, S. P., Weaver, J. C., \& Inciardi, J. A. (2005). The Connections of Mental Health Problems, Violent Life Experiences, and the Social Milieu of the "Stroll” with the HIV Risk Behaviors of Female Street Sex Workers. Journal of Psychology \& Human Sexuality, 17(1-2), 23-44. doi:10.1300/J056v17n01_03

Turner, A. K., Latkin, C., Sonenstein, F., \& Tandon, S. D. (2011). Psychiatric disorder symptoms, substance use, and sexual risk behavior among African-American out of school youth. Drug \& Alcohol Dependence, 115(1-2), 67-73. 
doi:10.1016/j.drugalcdep.2010.10.012

UNAIDS. (2011). A New Investment Framework for the Global HIV Response. Retrieved from Geneva:

UNAIDS. (2013). Global Report: UNAIDS report on the global AIDS epidemic 2013. Retrieved from Geneva:

Urada, L. A., Morisky, D. E., Hernandez, L. I., \& Strathdee, S. A. (2013). Social and structural factors associated with consistent condom use among female entertainment workers trading sex in the Philippines. AIDS Behav, 17(2), 523-535.

Vanwesenbeeck, I. (2001). Another Decade of Social Scientific Work on Sex Work: A Review of Research 1990-2000. Annual Review of Sex Research, 12, 242. Retrieved from http://search.ebscohost.com/login.aspx?direct=true\&db=aph\&AN=6324824\&site =ehost-live

Weinhardt, L. S., Carey, M. P., Carey, K. B., Maisto, S. A., \& Gordon, C. M. (2001). The relation of alcohol use to HIV-risk sexual behavior among adults with a severe and persistent mental illness. Journal of Consulting and Clinical Psychology, 69(1), 77-84. Retrieved from http://search.proquest.com/docview/614368103?accountid=14548

Weinhardt, L. S., Carey, M. P., Carey, K. B., \& Verdecias, R. N. (1998). Increasing assertiveness skills to reduce HIV risk among women living with a severe and persistent mental illness. Journal of Consulting and Clinical Psychology, 66(4), 680-684. doi:10.1037/0033-2909.111.3.455

10.1037/0022-006x.66.4.680

Wong, W., Holroyd, E., \& Bingham, A. (2011). Stigma and sex work from the perspective of female sex workers in Hong Kong. Sociology of Health \& Illness, 33(1), 50-65. doi:10.1111/j.1467-9566.2010.01276.x

Wong, W., Holroyd, E., Gray, A., \& Ling, D. C. (2006). Female street sex workers in Hong Kong: Moving beyond sexual health. Journal of Women's Health, 15(4), 390-397.

Yi, H., Mantell, J. E., Wu, R., Lu, Z., Zeng, J., \& Wan, Y. (2010). A profile of HIV risk factors in the context of sex work environments among migrant female sex workers in Beijing, China. Psychology, Health \& Medicine, 15(2), 172-187. doi:10.1080/13548501003623914

Zhang, C., Li, X., Hong, Y., Chen, Y., Liu, W., \& Zhou, Y. (2012). Partner Violence and HIV Risk Among Female Sex Workers in China. AIDS Behav, 16(4), 1020-1030. doi:10.1007/s10461-011-9968-0 